\title{
Osteomalacia tumoral. Reporte de un caso y revisión de la literatura
}

\author{
Luis Felipe Fierro-Maya ${ }^{1}$, Héctor Sandoval ${ }^{2}$ Liliana Mejía ${ }^{3}$, Óscar Messa-Botero ${ }^{4}$, Mario \\ Alexánder Melo-Uribe ${ }^{5}$, Camilo Soto Montoya ${ }^{6}$, Andrés Arturo Cuellar ${ }^{1}$, Mireya Tapiero ${ }^{1}$
}

\author{
${ }^{1}$ Endocrinología oncológica, Instituto Nacional de Cancerología, \\ Bogotá, Colombia. \\ ${ }^{2}$ Endocrinólogo, Profesor Facultad de Medicina, Universidad \\ Nacional de Colombia. \\ ${ }^{3}$ Endocrinóloga, Clinicentro Sanitas EPS, Bogotá, Colombia. \\ ${ }^{4}$ Patólogo oncólogo, Instituto Nacional de Cancerología, Bogotá, \\ Colombia. \\ ${ }^{5}$ Patología, Universidad de la Sabana. \\ ${ }^{6}$ Ortopedista oncólogo, coordinador de la Clínica de Ortopedia \\ Oncológica, Instituto Nacional de Cancerología, Bogotá, Colombia. \\ Correspondencia: Luis Felipe Fierro Maya, coordinador en- \\ docrinología oncológica del Instituto Nacional de Cancerolo- \\ gía, Calle 1 \# 9-85, Bogotá, Colombia. \\ Correo electrónico: ffierro@cancer.gov.co \\ Fecha de recepción: 27/07/2017 \\ Fecha de aceptación: 1/09/2017
}

\section{Introducción}

La osteomalacia tumoral (OT) es un síndrome paraneoplásico poco frecuente caracterizado por una alteración en la tasa de reabsorción del fósforo a nivel renal, causado por la producción tumoral de la proteína fosfatúrica, llamada factor de crecimiento fibroblástico 23 (FGF-23). Las manifestaciones clínicas son consecuencia de los niveles bajos de fósforo sérico que llevan a dolor musculoesquelético y fracturas por fragilidad ósea. Usualmente se trata de un tumor de origen mesenquimal de pequeño tamaño que puede ser difícil de localizar, pero en los casos en los que se logra realizar la extirpación quirúrgica del mismo, los síntomas y las anomalías bioquímicas revierten rápidamente ${ }^{(1)}$.

Presentamos un caso de un paciente con un tumor de gran tamaño y una enfermedad de larga evolución, que tuvo un desenlace exitoso luego de la cirugía.

\section{Caso clínico}

Se trata de un hombre de 56 años, con cuadro crónico de dolor en miembro inferior derecho progresivo, que inició hace aproximadamente dos años, hasta limitarlo en la marcha en los últimos dos meses, asociado a la presencia de una masa en la tibia derecha, de lento crecimiento, notada por el paciente desde hace al menos 12 meses. Como antecedentes, tuvo una fractura en fémur izquierdo 18 meses antes de la consulta, por un trauma menor, que fue tratada con cirugía de osteosíntesis y colocación de clavo intramedular.

Al examen físico con signos vitales normales, buen estado general y lo único relevante fue la presencia de una masa sobre la región anterior del tercio proximal de la tibia derecha, de aproximadamente $8 \times 10 \mathrm{~cm}$ de consistencia pétrea (figura 1).

Se le realizaron imágenes por resonancia magnética de la pierna derecha, encontrando una masa de aspecto neoplásico, como se describe en la figura 1.

En la búsqueda de más lesiones tumorales se practicaron imágenes por tomografía computarizada de tórax y de abdomen, las cuales fueron normales. La gammagrafía ósea mostró múltiples lesiones focales, descritas en la figura 2, que inicialmente fueron descritas como lesiones metastásicas, pero luego, tras la revaloración del caso a la luz de las pruebas bioquímicas, unas lesiones fueron interpretadas como zonas de pseudorreactivación de las placas de crecimiento, otras como fracturas antiguas y la imagen en la tibia derecha, interpretada como lesión tumoral.

Las pruebas bioquímicas revelaron hipofosfatemia persistente con una tasa de reabsorción tubular de fósforo también disminuida. Los valores de calcio sérico y de hormona paratiroidea intacta estuvieron en rangos de la normalidad, como se resume en la tabla 1 . Se evaluaron los niveles de factor de crecimiento fibroblástico 23 (FGF-23) en suero, con un resultado elevado más de tres veces por encima del valor de referencia.

Se hizo corrección de los niveles séricos de fosfato mediante infusión intravenosa en dos oportunidades durante su estancia hospitalaria, se dio suplementación de vitamina D3 (colecalciferol) por vía oral y se llevó a cirugía por parte del servicio de ortopedia, realizándose extirpación de la masa tibial derecha y osteosíntesis de la fractura de la tibia (figura 3). El reporte de patología informó compromiso por una neoplasia mesenquimal de histología benigna, como se muestra en la figura 4.

Luego de la cirugía, los niveles de fósforo sérico mejoraron de manera progresiva, sin necesidad de nuevas infusiones in- 
Figura 1. A. Fotografía de la lesión tumoral de $10 \mathrm{~cm}$ de diámetro en la porción proximal de la pierna derecha del paciente. B y C. Imágenes por resonancia magnética de la pierna derecha en las que se ve una masa de aspecto neoplásico (flecha blanca), dependiente de la cortical anterior de la tibia con crecimiento hacia tejidos blandos y escaso crecimiento hacia la cavidad endomedular, múltiples fracturas trabeculares por insuficiencia en tibia y peroné.

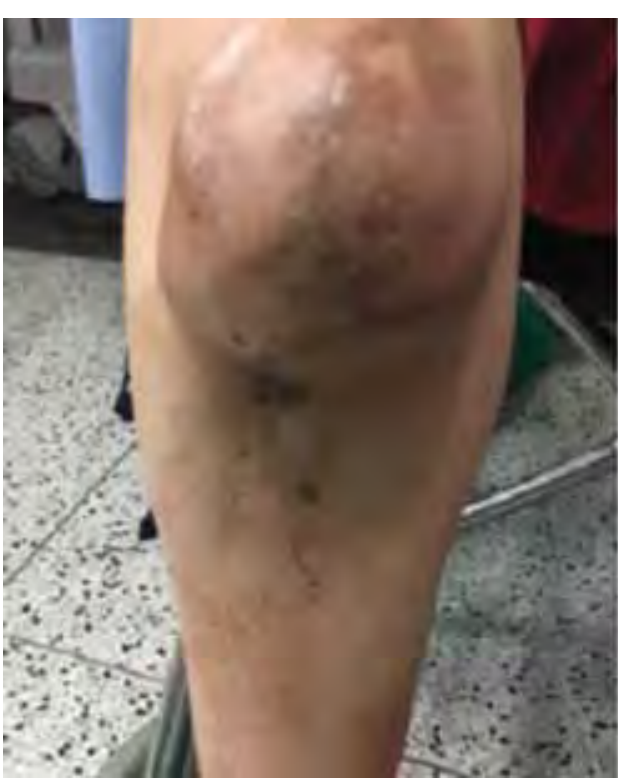

travenosas de fosfato. Tres semanas después de la cirugía se tomó una nueva muestra para determinar los niveles séricos de FGF-23, los cuales descendieron de manera significativa (tabla 1). Con los hallazgos y la evolución mencionada se confirmó el diagnóstico de osteomalacia inducida por tumor secundaria a un tumor mixto del tejido conectivo.

\section{Discusión}

El síndrome paraneoplásico de OT se asocia a tumores de origen mesenquimal generalmente benignos que se caracterizan histológicamente por presencia de células gigantes, fibromas osificantes, osteoblastomas, granulomas o hemangiopericitomas, que pueden localizarse casi que en cualquier parte del cuerpo, pero se han descrito con mayor frecuencia en las extremidades ${ }^{(2,3)}$.

El caso presentado ilustra la evolución usual de un paciente con osteomalacia tumoral, en quienes debido al desconocimiento de la enfermedad, el tiempo transcurrido desde la instauración de los síntomas hasta el diagnóstico, usualmente es prolongado ${ }^{(2)}$.

Si bien, la mayoría de casos describen que los tumores mesenquimales secretores de FGF-23 son pequeños y de difícil localización $^{(3,4)}$, el paciente que presentamos tenía
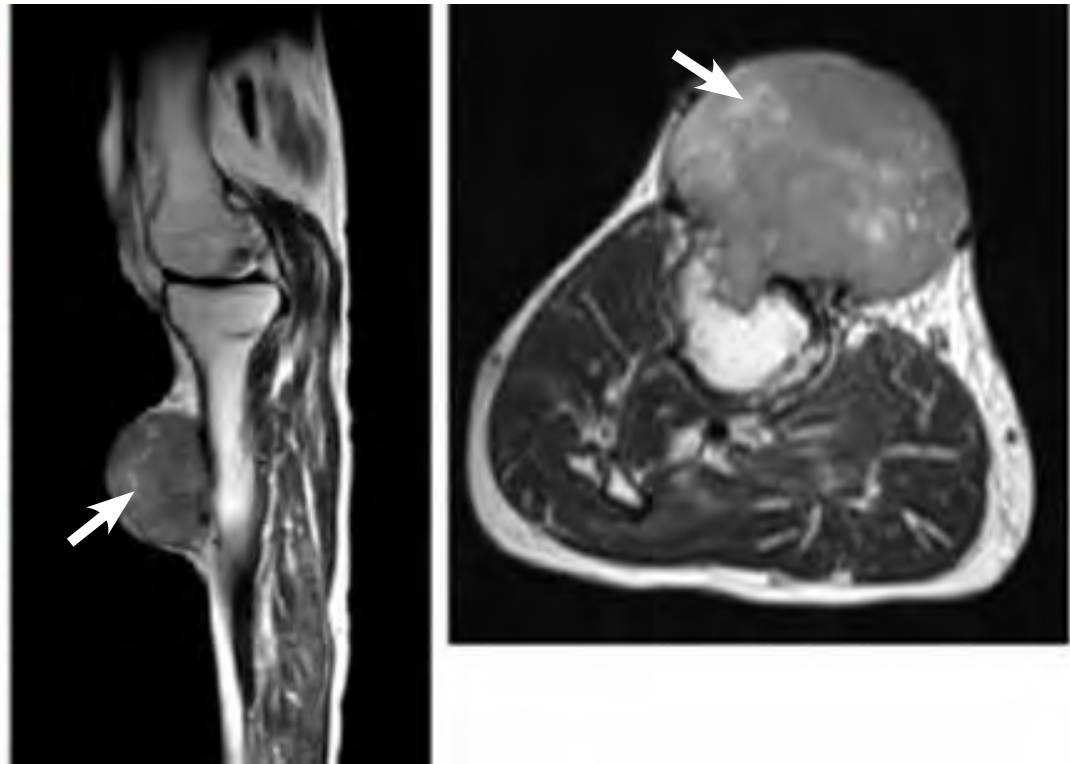

Figura 2. Gammagrafía ósea del paciente que muestra múltiples captaciones focales y alineadas en arcos costales, correspondientes a fracturas antiguas (flecha azules). Lesiones hipercaptantes en: diáfisis de cúbito derecho, en metadiáfisis de radio derecho, en tercio medio de la diáfisis del cúbito o radio izquierdo, en rama iliopúbica bilateral, en diáfisis proximal del fémur derecho, en diáfisis proximal del fémur izquierdo, fractura en tercio distal del fémur izquierdo (flechas negras). Incremento difuso de actividad de baja intensidad en el tercio proximal de la tibia derecha (flechas grises).

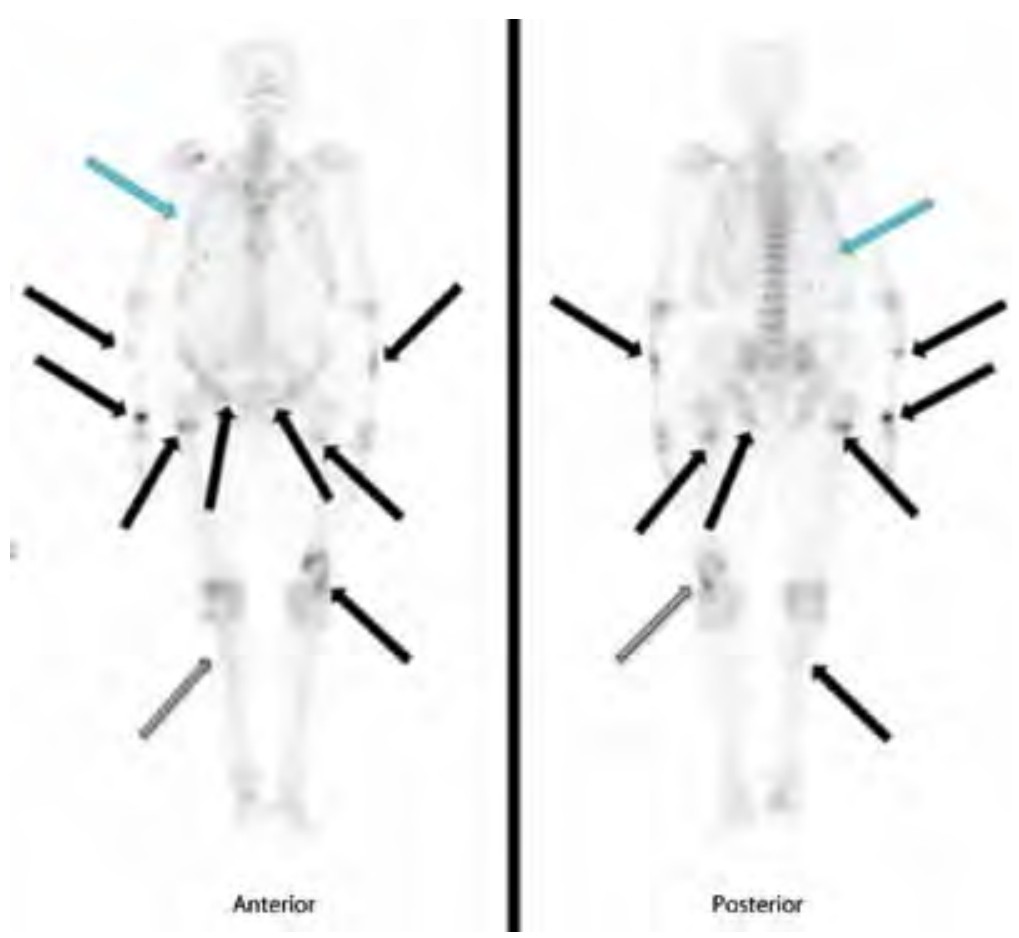


Tabla 1. Resultados de las pruebas de laboratorio clínico

\begin{tabular}{l|c|c}
\hline & Antes de cirugía & Después de cirugía \\
\hline Albúmina, g/dL & 4,1 & 4,0 \\
\hline $\begin{array}{l}\text { Calcio sérico, mg/dL } \\
\text { (VR 8,5-10,2) }\end{array}$ & 9,3 & 8,6 \\
\hline $\begin{array}{l}\text { Fósforo sérico, mg/dL } \\
\text { (VR 2,5-4,5) }\end{array}$ & 1,6 & 4,2 \\
\hline $\begin{array}{l}\text { Creatinina mg/dL } \\
\text { (VR 0,5-0,9) }\end{array}$ & 0,5 & 0,5 \\
\hline $\begin{array}{l}\text { PTH, pg/mL } \\
\text { (VR 15-65) }\end{array}$ & 68 & 56 \\
\hline $\begin{array}{l}\text { Vitamina D 25 OH, ng/mL } \\
\text { (VR 30-50) }\end{array}$ & 11,8 & 23,2 \\
\hline $\begin{array}{l}\text { Fosfatasa Alcalina, U/L } \\
\text { (VR }\end{array}$ & 519 & 382 \\
\hline $\begin{array}{l}\text { Calcio orina 24 horas, mg/24 h } \\
\text { (VR 100-300) }\end{array}$ & 216 & ND \\
\hline $\begin{array}{l}\text { Reabsorción tubular de fósforo, \% } \\
\text { (VR 85-95) }\end{array}$ & $77 \%$ & ND \\
\hline $\begin{array}{l}\text { TmP/FG, mg/dL } \\
\text { (VR 2,8-4,4) }\end{array}$ & 595 & Menor de 50 \\
\hline $\begin{array}{l}\text { FGF-23, Ensayo inmunométrico, RU/mL } \\
\text { (VR menos de 180) }\end{array}$ & 1,34 & ND \\
\hline VR:Var res & & \\
\hline
\end{tabular}

VR: Valor de referencia; TmP/FG: Reabsorción tubular máxima de Fósforo corregido por tasa de filtración glomerular; FGF-23: Factor de Crecimiento Fibroblástico 23; ND: no determinado.

Figura 3. A. Fotografías tomadas durante el procedimiento quirúrgico de extirpación de la masa y $B$. Imágenes de radiografía simple de pierna que muestra la oteosíntesis de la fractura
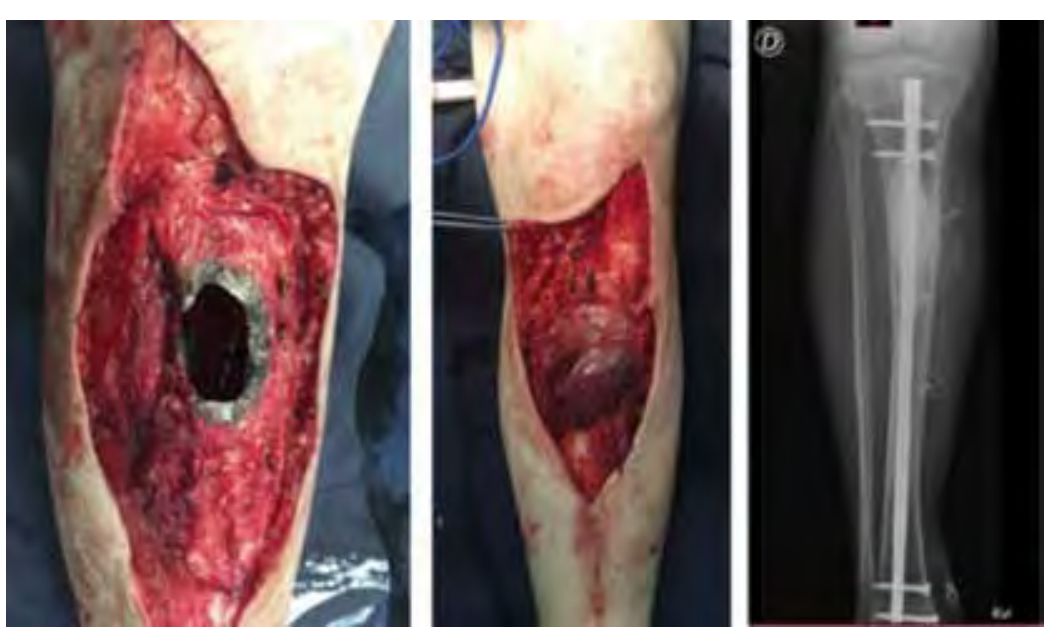

una masa tumoral de $10 \mathrm{~cm}$ y por ello no fueron necesarios estudios más avanzados para su localización, tales como los estudios funcionales con marcadores de receptores de somatostatina (gammagrafía con octreotide o tomografía por emisión de positrones con galio 68 - octreotide) o con tomografía por emi- sión de positrones con fluordesoxiglucosa. Algunos autores han reportado la poca utilidad de la gammagrafía ósea con tecnecio MIBI y la inducción de errores en curso de la localización de la lesión tumoral, como inicialmente ocurrió en nuestro caso, al interpretar las hipercaptaciones en las placas de crecimiento de las extremidades como zonas sospechosas de metástasis, que posiblemente corresponden a zonas de pseudorreactivación ${ }^{(1)}$.

Las manifestaciones clínicas musculares como mialgias, debilidad muscular proximal, el dolor óseo y las fracturas por insuficiencia, presentes en el paciente descrito, son causadas por la hipofosfatemia y la deficiencia de vitamina $\mathrm{D}$, por ello, ante un paciente con una historia clínica sugestiva, es fundamental el abordaje diagnóstico apropiado para un adecuado diagnóstico diferencial. En este sentido, se debe evaluar inicialmente el calcio sérico (ionizado o total corregido por el valor de albúmina sérica), fósforo sérico, magnesio sérico, niveles de hormona paratiroidea, niveles de 25 hidroxi vitamina D3 y creatinina sérica. Si se documenta hipofosfatemia, se debe proceder al cálculo de la tasa de reabsorción tubular de fósforo, usando la fórmula:

100x[1-((fosfato en orina/creatinina en orina) x (creatinina sérica/fosfato sérico)]

Normalmente se debe reabsorber entre el $85 \%$ y el $95 \%$ del fosfato, por tanto, encontrarlo disminuido nos debe llevar a buscar causas de pérdidas renales de fósforo, entre las cuales se cuentan: hiperparatirodismo primario, deficiencia de vitamina $\mathrm{D}$, enfermedades tubulares renales (hipofosfatemia ligada al X, síndrome de Fanconi, enfermedad de Dent, intolerancia a la fructosa, cetoacidosis diabética, acidosis metabólica y respiratoria, expansión de volumen y fármacos (calcitonina, diuréticos, glucocorticoides, bicarbonato, quimioterapia, antirretrovirales, aminoglucósidos, anticonvulsivantes, hierro parenteral), trasplante renal y osteomalacia tumoral ${ }^{(5)}$.

Bioquímicamente la osteomalacia oncogénica se confirma por los hallazgos de hipofosfatemia, disminución de la tasa de reabsorción tubular de fósforo, niveles elevados o inapropiadamente normales de FGF-23, niveles bajos o inapropiadamente normales de 1,25 dihidroxivitamina D, paratohormona normal o levemente elevada y fosfatasa alcalina elevada ${ }^{(6)}$. 
Figura 4. A y B muestra la periferia del tumor, con arquitectura pseudolobulada, y células estrelladas y alargadas dispuestas en un estroma mixoide. $C$, se identifican células redondas que recuerdan condroblastos, positivas para S100 en inmunohistoquímica D. (A, 4X; B-D 10X.) (A-C, hematoxilina-eosina. D, inmunohistoquímica S100)
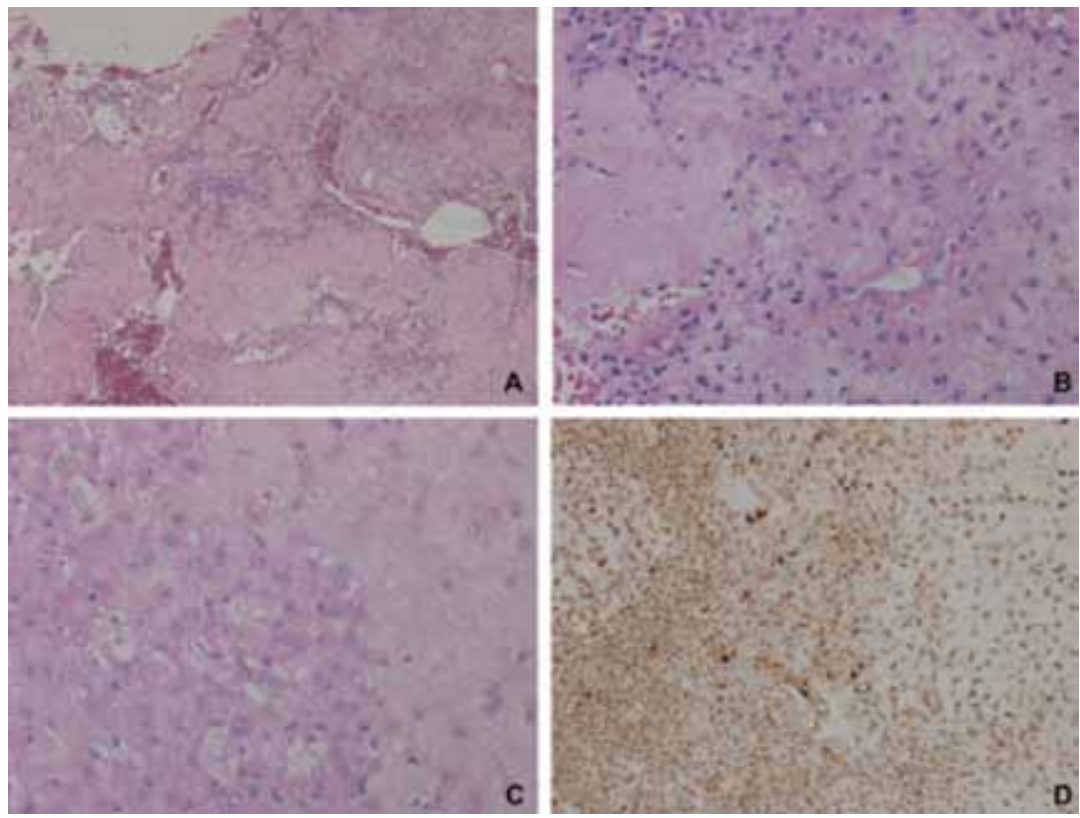

El FGF-23 normalmente es expresado en los osteocitos y regula el metabolismo del fósforo y la vitamina D mediante la unión al complejo receptor KlothoFGF. En el riñón disminuye la reabsorción tubular de fosfatos, inhibiendo la expresión de cotransportadores de sodio/fosfato tipo 2A y 2C e inhibe la actividad de la 1-alfa hidroxilasa renal $^{(7)}$.

La determinación de FGF-23 puede realizarse por diferentes técnicas analíticas. En el caso que presentamos se realizó en un laboratorio de referencia en Estados Unidos mediante un ensayo inmunométrico y se confirmó la caída de los niveles luego de la remoción quirúrgica del tumor. Para este método analítico, la sensibilidad ha sido reportada entre el $23 \%$ y el $86 \%{ }^{(2,8)}$.

El tratamiento de elección es la resección quirúrgica; sin embargo, ésta no siempre es posible dada la dificultad en la localización del tumor. En esos casos, se debe dar tratamiento médico con suplementos de fosfato y dosis altas de calcitriol $^{(1)}$. En los casos muy sintomáticos, con hipofosfatemia severa, como en nuestro caso, la reposición con fosfato intravenoso es mandatoria. Para ello se usa una infusión intravenosa de fosfato de potasio que se prepara con una ampolla de $10 \mathrm{ml}$ (que contiene fosfato dibásico de potasio $1.550 \mathrm{~g}$, fosfato monobásico de potasio $0.300 \mathrm{~g}$, potasio $20 \mathrm{mEq}$. Fosfato $20 \mathrm{mEq}^{(9)}$ diluida en 500 $\mathrm{ml}$ de solución glucosada al $5 \%$ o $500 \mathrm{ml}$ solución de cloruro de sodio al 0,9\%, a una dosis de 2,5 a $5 \mathrm{mg}$ por kilo de peso, y a una velocidad de infusión lenta, generalmente en no menos de 6 horas $^{(5)}$.

En los casos menos severos, se pueden utilizar preparaciones de fosfato para uso oral, que en nuestro medio pueden conseguirse en preparaciones magistrales en bolsas o sachet (que contienen fosfato de sodio bifásico anhidro $852 \mathrm{mg}$ + fosfato de potasio $155 \mathrm{mg}$ + fosfato de sodio monobásico monohidratado $45 \mathrm{mg}^{(10)}$.
Como se ha descrito en otros casos, las anomalías bioquímicas revierten rápidamente luego de la cirugía, es por ello, que a los pacientes en quienes no se logre la identificación del tumor responsable, se deben repetir las imágenes funcionales con intervalos de uno a dos años.

En conclusión, la presentación de este caso clínico nos permite hacer una revisión de la literatura sobre este raro trastorno endocrinológico y nos confirma que debemos estar alerta a los síntomas característicos del mismo, pues como pudo mostrarse, es posible en nuestro medio llegar a un diagnóstico acertado y a un tratamiento adecuado.

\section{Agradecimientos}

A la Asociación Colombiana de Endocrinología, Diabetes y Metabolismo y al Laboratorio de Investigación Hormonal, pues ambos hicieron posible las determinaciones de los niveles séricos de FGF-23

\section{Referencias}

1. William H Chong, Alfredo A Molinolo, Clara C Chen MTC. Tumor-Induced Osteomalacia. Endocr Relat Cancer. 2011;18(3):R53-77.

2. Alonso G, Varsavsky M. Osteomalacia tumoral : un síndrome paraneoplásico emergente. Endocrinol y Nutr. SEEN; 2016;63(4):181-6.

3. Folpe AL, Fanburg-smith JC, Billings SD, Reith JD Connell JXO, Rosenberg AE, et al. Most Osteomalacia-associated Mesenchymal Tumors Are a. Am J Surg Pathol. 2004;28(1):1-30.

4. P Andreopoulou, CM Millo, JC Reynolds, MH Kelly, BA Brillante, FM Wodajo, R Chang CC and MC. Multimodality Diagnosis and Treatment of Tumor-Induced Osteomalacia. Endocrine Reviews. 2010. p. (Suppl 1):0R08-6; S49.

5. Portillo MR. Nefrología al día. 2012th ed. Víctor Lorenzo JML-G, editor. Vol. 25. Madrid: Revista Nefrología; 2010. 201-219 p.

6. Nanes MS. Phosphate wasting and fibroblast growth factor-23. Curr Opin Endocrinol Diabetes Obes. 2013;20(6):523-31.

7. Hautmann AH, Hautmann MG, Kölbl O, Herr W, Fleck M. Tumor-Induced Osteomalacia: an Up-toDate Review. Curr Rheumatol Rep. 2015;17(6).

8. Hu F, Jiang C, Zhang Q, Shi H, Wei L, Wang Y. Quantitative ELISA-Like Immunohistochemistry of Fibroblast Growth Factor 23 in Diagnosis of TumorInduced Osteomalacia and Clinical Characteristics of the Disease. Dis Markers. 2016;2016.

9. http://co.prvademecum.com/producto. php?producto $=9551$ [Internet]. [cited 2017 Jun 20]. Available from: http://co.prvademecum.com/ producto.php?producto $=9551$

10. http://www.fagronorbus.com/productos-laboratorio-farmaceutico-magistral-colombia.html [Internet]. [cited 2017 Jun 27]. Available from: http:// www.fagronorbus.com/productos-laboratoriofarmaceutico-magistral-colombia.html 